\title{
Inflammatory bowel disease in pregnancy
}

\author{
Amelie I. Stritzke MD, Cynthia H. Seow MBBS(Hons)
}

Cite as: CMAJ 2017 May 8;189:E669. doi: 10.1503/cmaj.160967

1

In most women with inflammatory bowel disease (IBD), the condition is diagnosed during prime child-bearing years With 1 in 150 Canadians affected and more than half of them female, Canada has among the highest incidence and prevalence rates of IBD in the world. ${ }^{1}$

2

Active IBD (Crohn disease or ulcerative colitis) during conception and pregnancy increases the risk of adverse pregnancy outcomes Adverse outcomes include prematurity, intrauterine growth restriction, spontaneous abortion, stillbirth and neonatal death. ${ }^{2}$ Therefore, preconception care and regular review of disease activity by a gastroenterologist during pregnancy are important. ${ }^{3}$

Except for methotrexate, IBD therapies should be continued throughout pregnancy and lactation to opti mize and maintain disease control ${ }^{4}$

Women taking methotrexate should stop using it at least three months before attempting to conceive. ${ }^{4}$ Corticosteroids should be used with caution given the risk of gestational diabetes and hypertension. ${ }^{4} 5$-Aminosalicylic acid (5-ASA) compounds and azathioprine can be safely continued. ${ }^{4}$ To minimize transplacental transfer, the last dose of biologic therapies in pregnancy, including anti-tumour necrosis factor-alpha (anti-TNF- $\alpha$ ) therapy, should be provided in the middle of the third trimester and resumed immediately post partum. ${ }^{4}$ Because there is minimal transmammary transfer of IBD therapies, breastfeeding is encouraged. ${ }^{4}$

4.

Vaginal delivery should be considered in women with IBD unless they have active perianal disease or have undergone an ileoanal anastomosis ${ }^{4}$

Historically, population-based studies have shown that women with IBD are at higher risk of cesarean delivery than women in the general population in the absence of defined gastroenterologic or obstetric indications. ${ }^{4}$

Transplacental transfer of biologic therapies, specifically anti-TNF- $\alpha$ therapy, means that the live rotavirus vaccines scheduled at two and four months of age should be omitted ${ }^{5}$ All other live vaccines should be provided according to the Canadian Immunization Guide, including live measles-mumps-rubella and varicella vaccines at 12 months of age, because the anti-TNF- $\alpha$ drug will have cleared by then. ${ }^{5}$ Expert advice should be sought if other live vaccines (e.g., yellow fever and bacille Calmette-Guérin vaccines) are required before 12 months of age for travel indications.

\section{References}

1. The impact of inflammatory bowel disease in Canada: 2012 final report and recommendations. Toronto: Crohn's and Colitis Foundation of Canada; 2012. Available: www. isupportibd.ca/pdf/ccfc-ibd-impact-report-2012.pdf (accessed 2016 Nov. 1).

2. O'Toole A, Nwanne O, Tomlinson T. Inflammatory bowel disease increases risk of adverse pregnancy outcomes: a metaanalysis. Dig Dis Sci 2015;60:2750-61.

3. de Lima A, Zelinkova Z, Mulders AG, et al. Preconception care reduces relapse of inflammatory bowel disease during pregnancy. Clin Gastroenterol Hepatol 2016;14:1285-92.e1.

4. Nguyen GC, Seow CH, Maxwell C, et al.; IBD in Pregnancy Consensus Group; Canadian Association of Gastroenterology. The Toronto consensus statements for the management of inflammatory bowel disease in pregnancy. Gastroenterology 2016;150:734-57.e1.

5. Julsgaard M, Christensen LA, Gibson PR, et al. Concentrations of adalimumab and infliximab in mothers and newborns, and effects on infection. Gastroenterology 2016;151: 110-9.

Competing interests: The authors are involved in a research project supported by the MSI Foundation and the American College of Gastroenterology regarding improving clinical pathways in IBD care during pregnancy. Cynthia Seow has received honoraria as an advisory board member for Janssen Pharmaceutical, AbbVie, Shire, Actavis, Takeda Canada and Ferring Pharmaceuticals; speaker fees from Janssen Pharmaceutical and AbbVie; and research and educational support from Janssen Pharmaceutical and AbbVie. No other competing interests were declared.

This article has been peer reviewed.

Affiliations: Department of Pediatrics (Stritzke), Section of Neonatology; Department of Medicine and Community Health Sciences (Seow), Division of Gastroenterology and Hepatology, University of Calgary, Calgary, Alta.

Correspondence to: Amelie Stritzke, amelie.stritzke@albertahealthservices.ca 